\title{
THE WAVEFRONT INDUCED \\ IN A HOMOGENEOUSLY SHEARING SOLID BY A LOCALIZED MATERIAL IMPERFECTION*
}

BY

M. TOULIOS

Brown University.

\begin{abstract}
The shape of the two dimensional wavefront induced by a line material imperfection in a large body which is being subjected to a homogeneous, time dependent antiplane shear deformation, is investigated. The body is composed of isotropic, incompressible, hyperelastic material and the constitutive relation is assumed to be such that depending on the value of one parameter, strong ellipticity fails at a strain level corresponding to the local maximum of the shear stress-strain curve. The wavefront shapes are compared when this occurs and when it does not.
\end{abstract}

1. Introduction. The conditions which allow a homogeneous deformation to undergo an abrupt transition and give way to highly non-uniform deformations has been the subject of many studies in the past. In particular, regions of inhomogeneity play an important role in the initiation and subsequent development of such deformations. When a body is being subjected to high strain rates, one approach in one-dimensional analyses is the phenomenon of wave trapping which is encountered in one-dimensional plastic wave propagation when the shear tangent modulus becomes zero and strain levels propagate at zero speed. Erlich et al. [1] first used this idea to postulate an adiabatic shear band formation criterion, and more recently $\mathrm{Wu}$ and Freund [2], studied the mechanics of shear banding by considering a shear wave propagation in a half-space of a nonlinear material. A two-dimensional model of the localization of deformation during high rates of loading has been recently presented by $\mathrm{Wu}$ et al. [3]. They examined the evolution of a shear band which has been "triggered" by some material defect in the body. One of the conclusions of this work is that there are basic features in common with the concept of wave trapping.

In the present paper, a material imperfection is assumed to appear suddenly in a body of isotropic, incompressible and hyperelastic material which is undergoing a homogeneous and time dependent deformation in the antiplane shear mode. The chosen constitutive relation for the material is the one proposed by Knowles [4] and shows a local maximum

* Received April 27, 1984. 
in the dependence of shear stress on shear strain. In addition, at this maximum point there is a failure of both the ordinary and strong ellipticity of the corresponding equilibrium equations governing antiplane shear ([4] and Zee and Sternberg [5]). Assuming that the strength of the inhomogeneity is sufficiently small, the theory of small deformations superposed on large has been used in order to linearize the equation of motion about the state of the ongoing homogeneous strain. It is established that the influence of the defect appears as a disturbance inducing a transient deformation with elastic waves radiating outwards from the imperfect region. In particular here, a study is made of the wavefront shapes associated with a line material imperfection. It is found that as the level of the homogeneous deformation approaches the maximum in the shear stress-strain curve, the speed of the propagating wavefront goes to zero in a certain direction, therefore "trapping" the ensuing transient fields. The implications of the above are being discussed and comparisons are made to the work of $\mathrm{Wu}$ et al. [3].

An equivalent linearization procedure was first used by Abeyaratne and Triantafyllidis [6] in studying the emergence of shear bands from a material imperfection region in equilibrium, plane strain deformations as the imposed far field load is increased. Moreover, Payton [7] has applied the same formulation in order to investigate the wavefront shape induced in a homogeneously strained elastic body by a point perturbing body force.

2. Problem formulation. We consider a large body of isotropic, incompressible and hyperelastic material being subjected to a finite, homogeneous and time dependent antiplane shear deformation. A material particle initially at $X_{3}$ moves to $x_{3}$ with respect to a common rectangular Cartesian coordinate system. This is so positioned that the cross-section of the body spans the $\left(X_{1}, X_{2}\right)$ plane and the body is bounded by two planes at $X_{3}= \pm L$. In particular here, the homogeneous deformation is defined by the equations

$$
\begin{aligned}
& x_{1}=X_{1}, \\
& x_{2}=X_{2}, \\
& x_{3}=X_{3}+t\left(a X_{2}+b X_{1}\right),
\end{aligned}
$$

where $t$ is time and $a, b$ represent constant strain rates. This state of deformation of the body will be called $A$ and it can be easily seen that the field equations are identically satisfied.

The strain energy function $W$ per unit undeformed volume, is taken to be independent of the second invariant $I_{2}$ of the left Cauchy-Green tensor $\underline{B}$ and due to the assumption of material incompressibility,

$$
W=W\left(I_{1}\right)
$$

where $I_{1}=\operatorname{Tr} \underline{B}$ and $W\left(I_{1}\right)$ is twice continuously differentiable for $I_{1} \geqslant 3$. Moreover, according to the results of Knowles [8], the above restriction on $W$ satisfies the necessary and sufficient conditions on the strain energy function so that non-trivial finite states of antiplane shear exist.

At some time $t_{0}$ during the above deformation, a material imperfection appears in the body. Without any loss of generality, this will be taken as perturbing some material 
property by an amount $\varepsilon F(\underline{x})$, where $\varepsilon$ measures the strength of the imperfection. The consequence of this material inhomogeneity is to induce a further deformation in which a particle at $x_{3}$ is displaced to $\bar{x}_{3}$ and

$$
\bar{x}_{3}=x_{3}+\varepsilon u\left(x_{1}, x_{2}, t\right) .
$$

It is assumed that $\varepsilon$ is small in the sense that second and higher degrees in $\varepsilon$ may be neglected in comparison with those of first degree. The state of the deformation of the body, will now be denoted by $\bar{A}$.

We can now use the theory of the superposition of infinitesimal deformations on finite deformations, originally developed by Green et al. [9], in order to obtain linearized forms of the field equations. Initially, we quote some general results which can be cited in Eringen and Suhubi [10]. Let $s_{i j}(\underline{x}, t)$ be the components of the nominal stress tensor (i.e. the transpose of the Piola-Kirchhoff stress tensor), referred to the configuration of state $A$, when the body is in state $\bar{A}$. These are related to the components of $s_{i j}(\underline{X}, t)$, referred to the undeformed configuration, by the equation

$$
s_{i j}(\underline{x}, t)=\left(\partial x_{i} / \partial X_{k}\right) s_{k j}(\underline{X}, t) .
$$

The equations of motion for the body in state $\bar{A}$ when referred to the known state of homogeneous deformation are

$$
\partial s_{i j}(\underline{x}, t) / \partial x_{i}=\rho\left(\partial^{2} \bar{x}_{j} / \partial t^{2}\right)
$$

since no body forces are being applied.

As a result of the restriction (2.2) on the strain energy function and the occurred material imperfection, the components of the nominal stress tensor are now given by the constitutive relation

$$
s_{i j}(\underline{X}, t)=2 \phi\left(\partial \bar{x}_{j} / \partial X_{i}\right)-p\left(\partial X_{i} / \partial \bar{x}_{j}\right)
$$

where $\phi=W^{\prime}\left(I_{1}, X\right)=\partial W / \partial I_{1}$ and $p(\underline{X}, t)$ is an arbitrary scalar arising because of the incompressibility assumption. In accordance with (2.3), to first order in $\varepsilon, s_{i j}(\underline{X}, t)$, $p(\underline{X}, t)$ and $\phi$ at state $\bar{A}$ can be expanded as

$$
\begin{gathered}
s_{i j}(\underline{X}, t)=s_{i j}^{o}(t)+\varepsilon \bar{s}_{i j}(\underline{X}, t), \\
p(\underline{X}, t)=p^{o}(t)+\varepsilon \bar{p}(\underline{X}, t), \\
\phi\left(I_{1}, \underline{X}\right)=\phi^{o}\left(I_{1}\right)+\varepsilon \bar{\phi}\left(I_{1}, \underline{X}\right),
\end{gathered}
$$

where the superscript ( $o$ ) is associated with variables measured in the state $A$ (the uniform, time dependent field), and (-) surmounts the corresponding increments. Equivalent expressions were also introduced by Abeyaratne and Triantafyllidis [6]. Substituting (2.3), $\left(2.7_{2}\right)$ and $\left(2.7_{3}\right)$ in (2.6) and comparing the result with $\left(2.7_{1}\right)$, gives an expression for the increment of stress $\bar{s}_{i j}(\underline{X}, t)$ :

$$
\bar{s}_{i j}(\underline{X}, t)=2 \phi^{o} \frac{\partial u_{j}}{\partial x_{k}} \frac{\partial x_{k}}{\partial X_{i}}+2 \bar{\phi} \frac{\partial x_{j}}{\partial X_{i}}+p^{o} \frac{\partial X_{i}}{\partial x_{k}} \frac{\partial u_{k}}{\partial x_{j}}-\bar{p} \frac{\partial X_{i}}{\partial x_{j}} .
$$


The equations of motion (2.5) can be similarly evaluated to leading order, to give

$$
\partial \bar{s}_{i j}(\underline{x}, t) / \partial x_{i}=\rho\left(\partial^{2} u_{j} / \partial t^{2}\right) \text {. }
$$

Finally, equation (2.8) can now be substituted in (2.9) on making use of an equivalent relation to (2.4) for the stress increments, and thus obtain the necessary equations for determining the perturbed transient deformation. It can be shown that for the antiplane shear mode these reduce to the single equation

$$
2 \phi^{\prime \prime}\left(u_{.11}+u_{.22}\right)+2 b t \bar{\phi}_{.1}+2 a t \bar{\phi}_{.2}=\rho u_{. t t}
$$

where (2.1) has also been used, and a subscript preceded by a comma indicates partial differentiation with respect to an $x_{i}$-coordinate or time. It can be seen that the equation (2.10) represents a linearized form of the field equations about the state of the ongoing homogeneous deformation.

3. Application to a specific material. In order to study the shape of the propagating wavefront associated with the material imperfection, we now proceed to consider a specific subclass of incompressible materials originally proposed by Knowles [4] and for which (2.2) holds. In addition, $W\left(I_{1}\right)$ satisfies the Baker-Ericksen inequality which here becomes $W^{\prime}\left(I_{1}\right)>0$ for $I_{1} \geqslant 3$. Let

$$
W\left(I_{1}\right)=\frac{\mu}{2 d}\left\{\left[1+\frac{d}{n}\left(I_{1}-3\right)\right]^{n}-1\right\}, \quad I_{1} \geqslant 3,
$$

where $\mu$ is the infinitesimal shear modulus, $n$ is a "hardening" parameter, $d$ a third material constant and all three are assumed to be positive. A complete description of this subclass of materials as $n$ varies can be found in Knowles [4], but here we limit the analysis to $n \leqslant 0.5$. The effect of this restriction is that curves of $(\tau-\gamma)$ have a well defined maximum for $n<0.5$ and $\tau \rightarrow 0$ as $\gamma \rightarrow \infty$, while for $n=0.5$ the maximum $\tau$ occurs at an infinitely large $\gamma$. The symbols $\tau$ and $\gamma$ denote measures of shear stress and shear strain respectively, and are defined by

$$
\tau=\sqrt{s_{13}^{2}+s_{23}^{2}}, \quad \gamma=\sqrt{u_{, \alpha} u_{, \alpha}},
$$

$u$ now referring to a general displacement, and here as well as throughout the paper, the Greek subscripts have the range 1,2. For the material defined by (3.1), $\tau$ and $\gamma$ are related by the following expression:

$$
\tau=2 \gamma W^{\prime}\left(I_{1}\right)=\mu\left(1+d \gamma^{2} / n\right)^{n-1} \gamma .
$$

The material imperfection will now be introduced as a perturbation to the infinitesimal shear modulus $\mu$. In particular, we consider a line imperfection at $x_{1}=x_{2}=0$ and $-L \leqslant x_{3} \leqslant L$; thus, at every cross-section of the body

$$
\mu=\mu^{\prime \prime}\left[1+\varepsilon \delta\left(x_{1}\right) \delta\left(x_{2}\right)\right]
$$

where $\mu^{\circ}$ denotes now the constant infinitesimal shear modulus of the material outside the defect. Using (3.1) and (3.4) the increment $\bar{\phi}$ can be determined by expanding $\phi$ at the state $\bar{A}$ using Taylor's series up to the first order in $\varepsilon$; hence,

$$
\begin{aligned}
\bar{\phi}= & \mu^{o}(n-1)(d / n)\left(a t u_{.2}+b t u_{.1}\right)\left[1+\left(a^{2}+b^{2}\right)\left(d t^{2} / n\right)\right]^{n-2} \\
& +(1 / 2) \delta\left(x_{1}\right) \delta\left(x_{2}\right)\left[1+\left(a^{2}+b^{2}\right)\left(d t^{2} / n\right)\right]^{n-1}
\end{aligned}
$$


Finally, substituting (3.5) into (2.10) gives the equation of motion for this particular material

$$
\begin{aligned}
A u_{.11} & +4(d / n)(n-1) a b t^{2} u_{.12}+B u_{.22}-c^{-2} D^{2-n} u_{. t t} \\
& =-\left(D / \mu^{o}\right) t f\left(x_{1}, x_{2}\right), \quad t>t_{0},
\end{aligned}
$$

where

$$
\begin{aligned}
A & =1-d(1-2 n) b^{2} t^{2} / n+d a^{2} t^{2} / n, \\
B & =1-d(1-2 n) a^{2} t^{2} / n+d b^{2} t^{2} / n, \\
D & =1+d\left(a^{2}+b^{2}\right) t^{2} / n=1+d \alpha^{2} t^{2} / n, \\
f\left(x_{1}, x_{2}\right) & =a \delta\left(x_{1}\right) \delta^{\prime}\left(x_{2}\right)+b \delta^{\prime}\left(x_{1}\right) \delta\left(x_{2}\right),
\end{aligned}
$$

and the dash in the Delta function denotes differentiation with respect to the argument inside the parenthesis. Moreover, $c=\left(\mu^{o} / \rho\right)^{1 / 2}$ is the speed of the infinitesimal elastic waves.

The second order partial differential equation (3.6) is linear with time dependent coefficients and as anticipated, it contains the influence of the defect as a source term. According to the theory of characteristics (for example see Courant and Hilbert [11]), a surface $S\left(x_{1}, x_{2}, t\right)=0$ is characteristic if the following condition is satisfied:

$$
A S_{.1}^{2}+4(d / n)(n-1) a b t^{2} S_{, 1} S_{.2}+B S_{.2}^{2}-c^{-2} D^{2-n} S_{. t}^{2}=0 .
$$

If the directions $n_{\alpha}$ and the speed $V$ are now introduced as

$$
\begin{aligned}
n_{\alpha} & =\left(\partial S / \partial x_{\alpha}\right) /\left(S_{. \beta} S_{. \beta}\right)^{1 / 2} \\
V & =(-\partial S / \partial t) /\left(S_{. \beta} S_{. \beta}\right)^{1 / 2}
\end{aligned}
$$

then $\underline{n}$ is the unit normal to the spatial characteristic curve $S=0$ and $V$ the corresponding speed of propagation. With this interpretation, equation (3.8) defines how the curve $S=0$ moves in $x_{\alpha}$-space. The substitution of (3.9) into (3.8) gives the following important form of the characteristic condition

$$
A n_{1}^{2}+4(d / n)(n-1) a b t^{2} n_{1} n_{2}+B n_{2}^{2}=c^{-2} D^{2-n} V^{2} .
$$

4. Conditions for strict hyperbolicity. In the previous section it was proved that the speed of propagation of each spatial characteristic curve $S=0$ in the direction of its normal $\underline{n}$ is given by (3.10). Following Jeffrey [12], the partial differential equation (3.6) will be said to be strictly hyperbolic if the second degree equation (3.10) in $V$ has two real and distinct roots for all choices of the unit vector $\underline{n}$.

We now proceed to determine the restrictions on the homogeneous deformation defined by $a t$ and $b t$, so that strict hyperbolicity is maintained. These can be easily determined if it is noticed that the effect of the term $b t X_{1}$ in $\left(2.1_{3}\right)$ is to rotate anticlockwise the principal axis of strain corresponding to the zero principal strain by an angle $\xi=\tan ^{-1}(-b / a)$. Hence if $\left(z_{\alpha}\right)$ is a new set of rectangular Cartesian coordinates so that the $z_{1}$-axis is aligned with this principal axis, equation (3.8) becomes

$$
D\left(\partial S / \partial z_{1}\right)^{2}+K\left(\partial S / \partial z_{2}\right)^{2}-c^{-2} D^{2-n}(\partial S / \partial t)^{2}=0
$$


where $D$ is defined in (3.7) and

$$
K=1-d(1-2 n) \alpha^{2} t^{2} / n .
$$

If we now define $\underline{\omega}=\left(\omega_{1}, \omega_{2}\right)$ as the unit normal to $S=0$ with reference to the $z_{\alpha}$ coordinates and then set $\omega_{1}=\cos \theta, \omega_{2}=\sin \theta$, where only $0 \leqslant \theta \leqslant \pi / 2$ need to be considered since (4.1) is a polynomial expression in $\cos ^{2} \theta$ and $\sin ^{2} \theta$, we obtain

$$
(V / c)^{2}=D^{n-2}\left(D \cos ^{2} \theta+K \sin ^{2} \theta\right)
$$

Since $D$ remains positive for all $t$, the speeds $V$ are always real and distinct if $K>0$, or

$$
\gamma^{2}=\left(a^{2}+b^{2}\right) t^{2}<n[d(1-2 n)]^{-1} .
$$

Comparison with equation (3.3) shows that (4.4) is equivalent to the requirement that the curve $\tau$ versus $\gamma$ has positive slope.

The reality of the roots $V$ is assured by the condition of strong ellipticity [10]. In general, strong ellipticity does not rule out repeated (nonzero) propagation speeds, but for this particular problem this situation does not arise. Therefore, the conditions of strict hyperbolicity and strong ellipticity are here equivalent. This can also be deduced from the results of [4] and [5], where it is proved that the restriction (4.4) is necessary and sufficient for the ordinary and strong ellipticity of the corresponding equilibrium equation. The implications of the failure of the above conditions on the wavefront propagation problem, are discussed in Sec. 6.

5. Wavefront construction. A cylindrical surface in the three-dimensional $\underline{x}$-space separating the homogeneous deformation from the perturbed motion represents the wavefront propagating away from the line source at the origin. It is well known that wavefronts are characteristic surfaces (Courant and Hilbert [11]). Therefore, in two dimensions it is convenient to specify the wavefront in the form

$$
S\left(x_{1}, x_{2}, t\right)=\sigma\left(x_{1}, x_{2}\right)-t=0 .
$$

The family of curves $\sigma\left(x_{1}, x_{2}\right)=t$, which are intersections of the cylindrical surfaces with the $\left(x_{1}, x_{2}\right)$ plane, give for increasing times the successive positions of the wavefront.

It is clear from the results of the previous section, that for any ratio $b / a$ the wavefront is symmetric with respect to the principal axis defined by the angle $\xi$. Thus, proceeding with the $z_{\alpha}$ coordinates, we can substitute (5.1) into (4.1) to obtain

$$
H\left(p_{\alpha}, z_{\alpha}, \sigma\right)=0
$$

where

$$
\begin{gathered}
H=\frac{1}{2} D p_{1}^{2}+\frac{1}{2} K p_{2}^{2}-\frac{1}{2} c^{-2} D^{2-n}, \\
p_{\alpha}=\partial \sigma / \partial z_{\alpha} .
\end{gathered}
$$

Equation (5.2) is a nonlinear first order partial differential equation for $\sigma$. The solution can be obtained from the following system of five ordinary differential equations (for example, see Whitham [13])

$$
\begin{aligned}
& d z_{1} / d \lambda=D p_{1}, \quad d z_{2} / d \lambda=K p_{2}, \\
& d p_{1} / d \lambda=-(d / n) \alpha^{2} t p_{1}^{3}+(d / n)(1-2 n) \alpha^{2} t p_{1} p_{2}^{2}, \\
& d p_{2} / d \lambda=-(d / n) \alpha^{2} t p_{1}^{2} p_{2}+(d / n)(1-2 n) \alpha^{2} t p_{2}^{3},
\end{aligned}
$$




$$
d \sigma / d \lambda=c^{-2} D^{2-n} .
$$

These determine a "characteristic" curve $z_{\alpha}(\lambda)$, also called a ray, and the values of $\sigma$ and $p_{\alpha}$ along it (where $\lambda$ is a suitable curve parameter). As it is clear from the equations (5.4), the rays are not normal to the wavefront. By multiplying $\left(5.5_{1}\right)$ by $p_{2}$ and $\left(5.5_{2}\right)$ by $p_{1}$ and subtracting, we obtain that along each ray the $p_{\alpha}$ 's are related by

$$
p_{1}=\beta p_{2}, \quad \beta=p_{1}^{o} / p_{2}^{o},
$$

where $p_{1}^{o}, p_{2}^{o}$ are the values of $p_{1}, p_{2}$ respectively, at the time $t=t_{0}$ when the material imperfection appears. The term $\beta$ can be found by considering the slope $m$ of the rays at the origin since the point source is situated there. Thus,

$$
m=d z_{1} / d z_{2}=\beta D\left(t_{o}\right) / K\left(t_{o}\right),
$$

and each value of $m$ determines a specific ray. Moreover, since $d H / d \lambda=0,(5.7)$ can be used in conjunction with (5.2) in order to calculate the value of $p_{2}$ (or $p_{1}$ ) along each ray. Hence,

$$
p_{2}^{2}=c^{-2} D^{2-n} /\left(D \beta^{2}+K\right) .
$$

Equations (5.4) can be normalized so that $\lambda$ represents distance measured along each ray. Further with this normalization, (5.6) is the directional derivative of the time of travel of the wavefront along the ray. Therefore, using (5.6), the coordinates of each ray specified by the initial slope $m$ are given by

$$
\begin{aligned}
& z_{1}(t)=c^{2} \beta \int_{t_{o}}^{t} D^{n-1}(t) p_{2}(t, \beta) d t, \\
& z_{2}(t)=c^{2} \int_{t_{o}}^{t} K(t) D^{n-2}(t) p_{2}(t, \beta) d t,
\end{aligned}
$$

and $p_{2}(t, \beta)$ is defined by (5.9). The integrands in (5.10) are well behaved functions and the coordinates of points along each ray at successive times $t$, can be easily found using a composite trapezoid numerical integration scheme. The propagating wavefront is then determined as contours of points at constant time $t$.

6. Results and discussion. The results of the previous section have been used to obtain detailed plots of the propagating wavefront and the corresponding rays. The chosen values for the material parameters are: $d=20,000, c=3,000 \mathrm{~m} / \mathrm{sec}$ and $n=0.4,0.5$. Without loss of generality, the strain rate $b$ is set equal to zero while $a=2,000 \mathrm{sec}^{-1}$, and therefore the wavefront is symmetric with respect to the $x_{\alpha}$-axes. Furthermore, the critical time at which the maximum of the shear stress-strain curve is reached in accordance with (4.4), is given by $t_{c r}=0.5 \times 10^{-5} \mathrm{sec}$.

Figure 1 shows the wavefront propagating in the homogeneously deformed material with the "hardening" exponent 0.4 , and associated with the line imperfection appearing at $t_{o}=0.3 t_{c r}$. The rays are chosen with a $10^{\circ}$ angle interval for the slope at the initial time $t_{o}$. The chosen times are expressed as fractions of the critical time, that is, $\eta=t / t_{c r}$. The effect of the failure of strong ellipticity is clearly shown in the $x_{2}$-direction where the wavefront appears to stop propagating as $\eta$ approaches one. This is also evident from (4.3) 


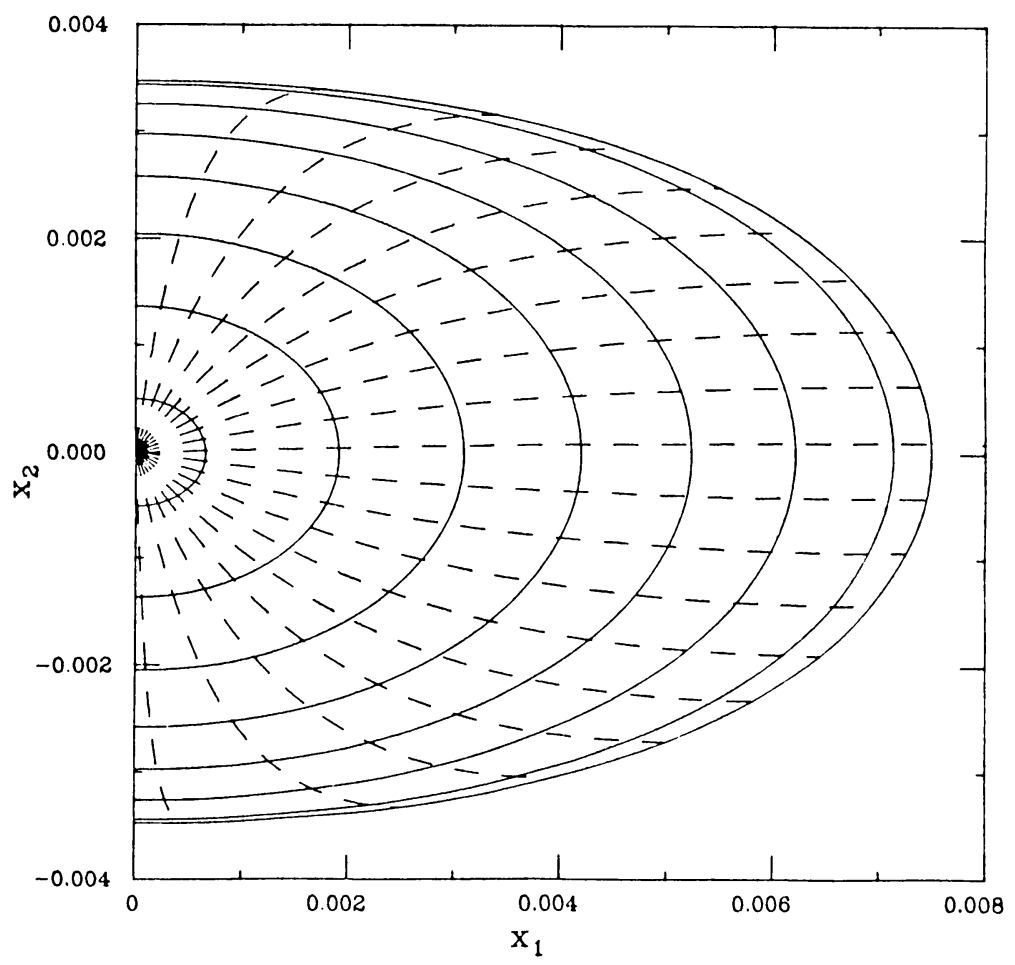

FIG. 1. Propagating wavefront at times $\eta=0.35(0.1) 0.95,0.99$ and rays for the material with $n=0.4$.

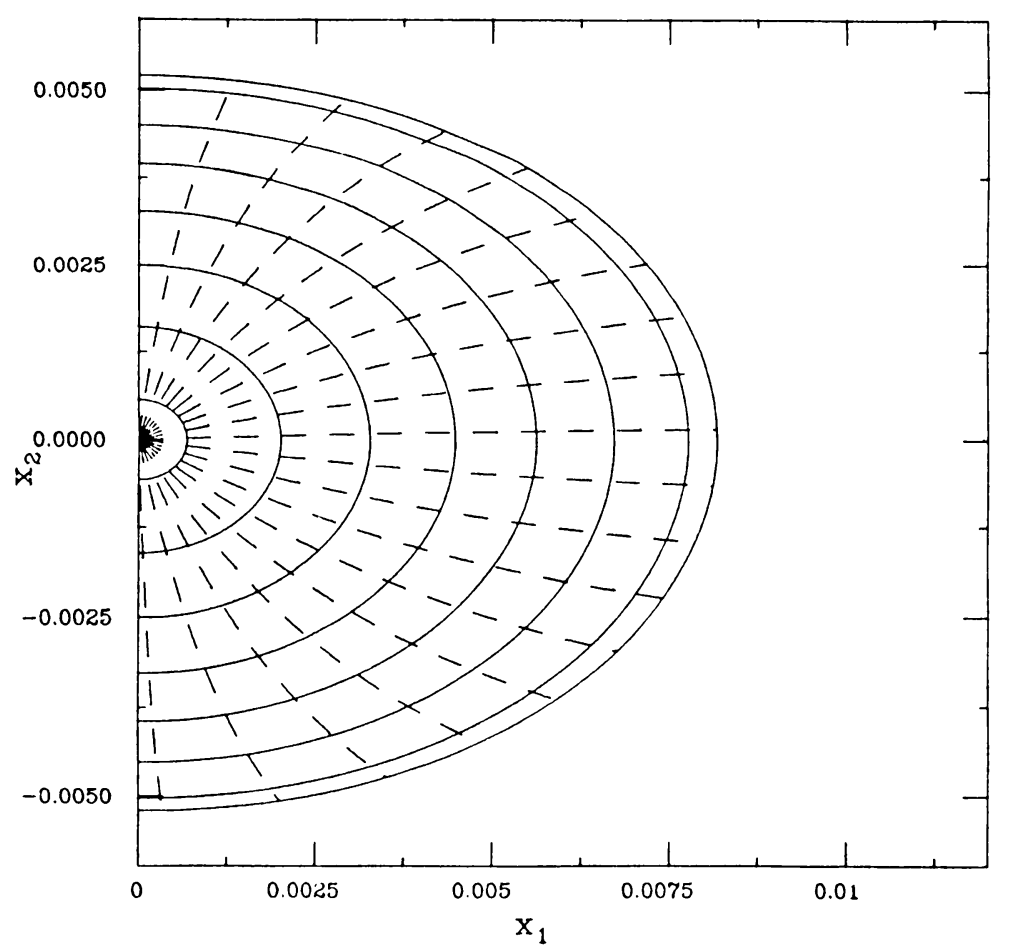

FiG. 2. Propagating wavefront at times $\eta=0.35(0.1) 0.95,0.99$ and rays for the material with $n=0.5$. 


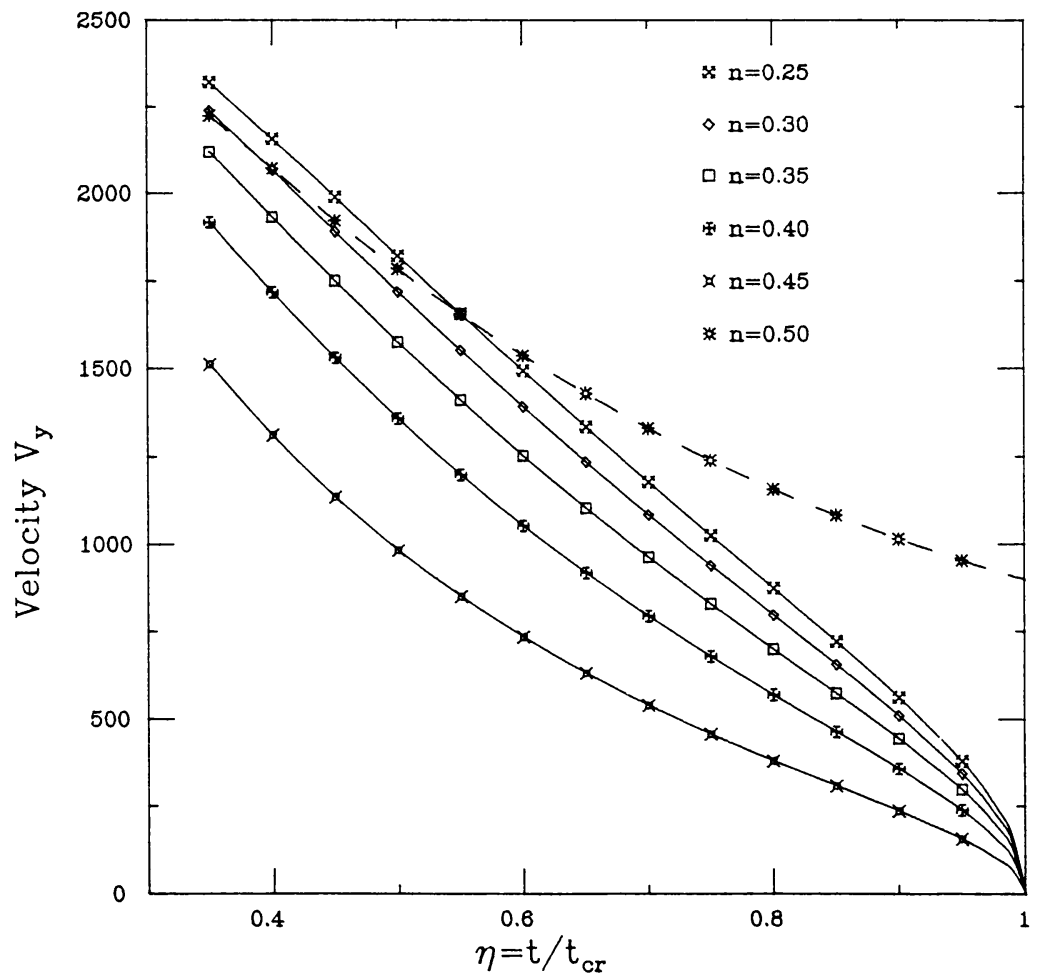

FIG. 3. Speed of propagation $V_{v}$ versus time $\eta$ for various hardening parameters.

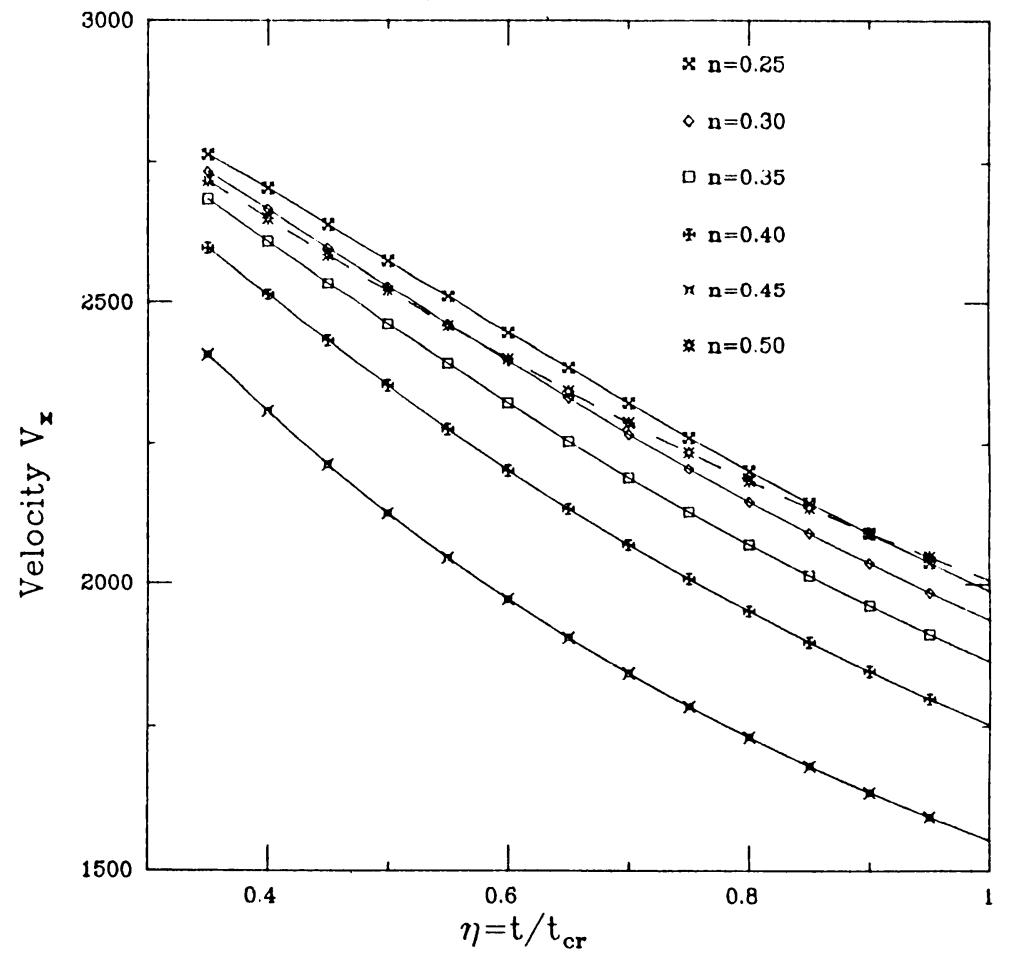

FIG. 4. Speed of propagation $V_{x}$ versus time $\eta$ for various hardening parameters. 
with $\theta=\pi / 2$, which shows that the speed of propagation first becomes zero and subsequently imaginary in this direction. If we make the unlikely assumption that the strength of the defect is sufficiently small as not to cause a local deformation effect, then as $t$ approaches the critical time, the wavefront appears to be "trapping" in the $x_{2}$-direction the ensuing transient fields. Subsequently, as a result of attaining strain levels corresponding to the part of the $(\tau-\gamma)$ curve with negative slope, the infinitesimal disturbances will grow large enough to destroy the background deformation. These remarks, which indicate the instability of the deformation, are made in analogy to the corresponding homogeneous equation of (3.6), which for a constant background strain can admit plane waves as generating the perturbed transient fields. The propagation of such plane waves has been studied by Hayes and Rivlin [14].

In reality, the inhomogeneity will concentrate the deformation in its vicinity and initiate a localized zone, which subsequently grows as a result of a strain concentrator at its edge. Such conclusions are supported by the numerical solution of the full nonlinear problem by Wu et. al. [3], where it was also found that the shear band grows at strain levels which are substantially below the critical level for the material outside the imperfect region (the specific instant of initiation depending on the strength of the defect).

When the "hardening" exponent is 0.5 , the $(\tau-\gamma)$ curve has no well defined maximum. The wavefronts are shown in Fig. 2, where the critical time is taken to be that of $n=0.4$ (for $n=0.5, t_{c r}=\infty$ ). Clearly, the superposed infinitesimal deformation will continue spreading in all directions (cf. (4.4) where now $\gamma<\infty$ ).

The speeds of propagation of the wavefront in the $x_{2}$ and $x_{1}$ directions, $V_{y}$ and $V_{x}$ respectively, can be obtained from (4.3) for $\theta=0$ and $\theta=\pi / 2$. Figs. 3 and 4 show the variation of these speeds at different times $\eta$ for a range of exponents $n$. As above, the $t_{c r}$ for $n=0.5$ is that of 0.4 and therefore, as $t_{\text {cr }}$ increases the curves corresponding to 0.5 translate towards the $\eta$-axis. Again, the concept of "wave trapping" for $n<0.5$ is apparent from Fig. 3 where $V_{v}=0$ at $\eta=1$.

Acknowledgements. I wouid like to express my appreciation to Professor L. B. Freund for bringing to my attention this problem. The research support of the U.S. Army Research Office under Grant DAAG-29-81-K-0121/6 with Brown University is gratefully acknowledged.

The computations were performed on the VAX11/780 Engineering Computer Facility at Brown University. This Facility was made possible by grants from the National Science Foundation (Solid Mechanics Program), the General Electric Foundation, and the Digital Equipment Corporation.

\section{REFERENCES}

[1] D. C. Erlich, D. R. Curran and L. Seaman, Further developments of a computational shear band model, SRI Rept. AMMRC TR 80-3 (1980)

[2] F. H. Wu and L. B. Freund, Deformation trapping due to thermoplastic instability in one-dimensional wate propagation. J. Mech. Phys. Solids 32, 119-132 (1984)

[3] F. H. Wu, M. Toulios and L. B. Freund, Initiation and propagation of shear bands in antiplane shear deformation, Brown University Technical Report, March 1984 
[4] J. K. Knowles, The finite antiplane shear field near the tip of a crack for a class of incompressible elastic solids, Intl. J. of Fracture 13, 611-639 (1977)

[5] L. Zee and E. Sternberg, Ordinary and strong ellipticity in the equilibrium theory of incompressible hyperelastic solids, Arch. Rational Mech. Anal. 83, 53-90 (1983)

[6] R. Abeyaratne and N. Triantafyllidis, The emergence of shear bands in plane strain, Int. J. Solids Structures 17 $1113-1134$ (1981)

[7] R. G. Payton, Two dimensional wavefront shape induced in a homogeneously strained elastic body by a point perturbing body force, Arch. Rational Mech. Anal. 32, 311-330 (1969)

[8] J. K. Knowles, On finite antiplane shear for incompressible elastic materia!s, J. Austral. Math. Soc. Ser. B, 19. 400-415 (1976)

[9] A. E. Green, R. S. Rivlin and R. T. Shield, General theory of small elastic deformations superposed on finite elastic deformations, Royal Society of London, Proceedings Ser. A., 211, 128-154 (1952)

[10] A. C. Eringen and E. S. Suhubi, Elastodynamics, Vol. I, Academic Press, New York, 1974

[11] R. Courant and D. Hilbert, Methods of mathematical physics, Vol. II, Interscience, New York, 1962

[12] A. Jeffrey, Quasilinear hyperbolic systems and waves, Pitman Publishing, London, 1977

[13] G. B. Whitham, Linear and nonlinear waves, John Wiley \& Sons, New York, 1974

[14] M. Hayes and R. S. Rivlin, Propagation of a plane wave in an isotropic elastic material subjected to pure homogeneous deformation, Arch. Rational Mech. Anal. 8, 15-22 (1961) 